
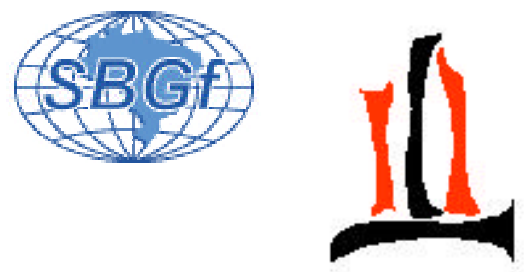

São Paulo 2004

\title{
Interpretação magnética-estrutural do embasamento da Bacia Bauru (Ks) no noroeste do estado do Paraná
}

Daniel Fabian Bettú ${ }^{1}$, Francisco José Fonseca Ferreira ${ }^{2},{ }^{1}$ Curso de Pós-Graduação em Geologia - UFPR, ${ }^{2}$ Laboratório de Pesquisas em Geofísica Aplicada - UFPR.

Copyright 2004, SBGf - Sociedade Brasileira de Geofísica

Este texto foi preparado para a apresentação no I Simpósio Regional da Sociedade Brasileira de Geofísica, São Paulo, 26-28 de setembro de 2004. Seu conteúdo foi revisado pela Comissão Tecno-científica do I SR-SBGf mas não necessariamente revisado pela Coñissão TeCno-cientifica do I SR-SBGr mas não necessariamente parcial deste material para propósitos comerciais sem prévia autorização da SBGf.

\section{Abstract}

The aim of this work was to build a magnetic-structural model of the basement of the Bauru Basin (Upper Cretaceous) in the northwest of the state of the Paraná, consisting of the basalts of the Serra Geral Formation, based in magnetic airbone data processing proceeding from the Projeto Aerogeofísico Rio Ivaí (Airbone Geophysical Rio Ivaí Project. The adoption of the inquiry method if gave because the basalts of the Serra Geral Formation are strong magnetic, contrasting with the sandstones of the Caiuá Group (Bauru Basin), inert to the terrestrial magnetic field. Finally structural-magnetic alignments of the basement with corresponding structures in the surface of the sandstones had been identified.

\section{Introdução}

A área deste estudo está localizada entre as coordenadas $23^{\circ} 00^{\prime}$ e $24^{\circ} 00^{\prime}$ de latitude sul e $52^{\circ} 30^{\prime}$ e $54^{\circ} 00^{\prime}$ de longitude oeste (Figura 1), no noroeste do estado do Paraná e exibe afloramentos dos arenitos de origem eólica do Grupo Caiuá (Bacia Bauru - Cretáceo Superior, Fernandes 1992).

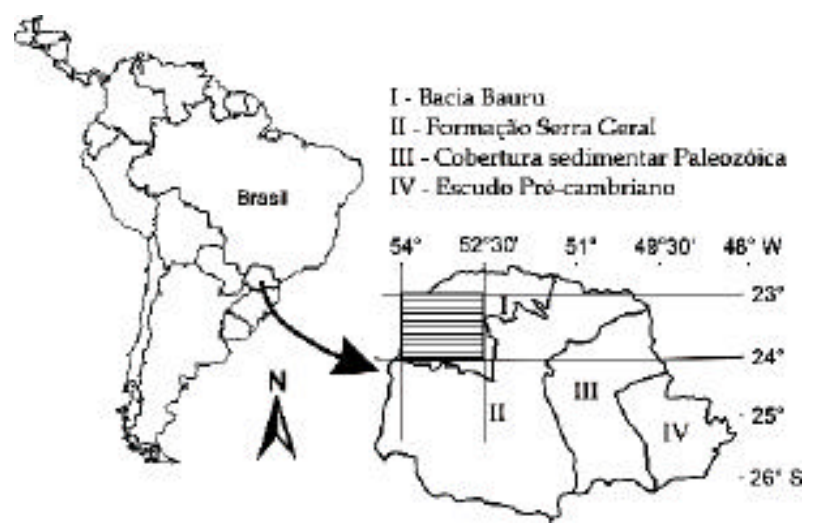

Figura 1 - Mapa de localização da área de estudo.

Estas rochas encontram-se depositadas sobre os basaltos da Formação Serra Geral (Cretáceo inferior), através de uma discordância erosiva, e por apresentar em sua composição mineralógica basicamente quartzo e microclínio, inertes ao campo magnético terrestre. O forte contraste entre a susceptibilidade magnética dos basaltos do embasamento e dos arenitos, aliada a pequena espessura do Grupo Caiuá (cerca de 270 metros), permitiram o processamento dos dados magnéticos, resultando na elaboração de um modelo estruturalmagnético do embasamento da Bacia Bauru. Este modelo foi posteriormente confrontado com as estruturas identificadas na superfície do Grupo Caiuá, resultando em um modelo estrutural integrado para a Bacia Bauru no noroeste do estado do Paraná.

\section{Métodos}

Os dados aeromagnéticos utilizados neste trabalho são provenientes do Projeto Aerogeofísico Rio Ivaí (Petrobrás 1980), adquiridos com linhas de vôo na direção N-S, espaçadas de $2000 \mathrm{~m}$, linhas de controle a cada 20000 $\mathrm{m}$, com altura de vôo constante de $450 \mathrm{~m}$ e com intervalo de amostragem de $56 \mathrm{~m}$. Esta base de dados foi interpolada através do algoritmo da curvatura mínima (Briggs 1974), para a geração de uma malha regular com células de 500 metros (1/4 do espaçamento entre as linhas de vôo). A malha gerada foi então subtraída do campo magnético da Terra (IGRF - International Geomagnetic Reference Field) e micronivelada através do método de decorrugação FFT (Fast Fourier Trasform), resultando no mapa mostrado na Figura 2.

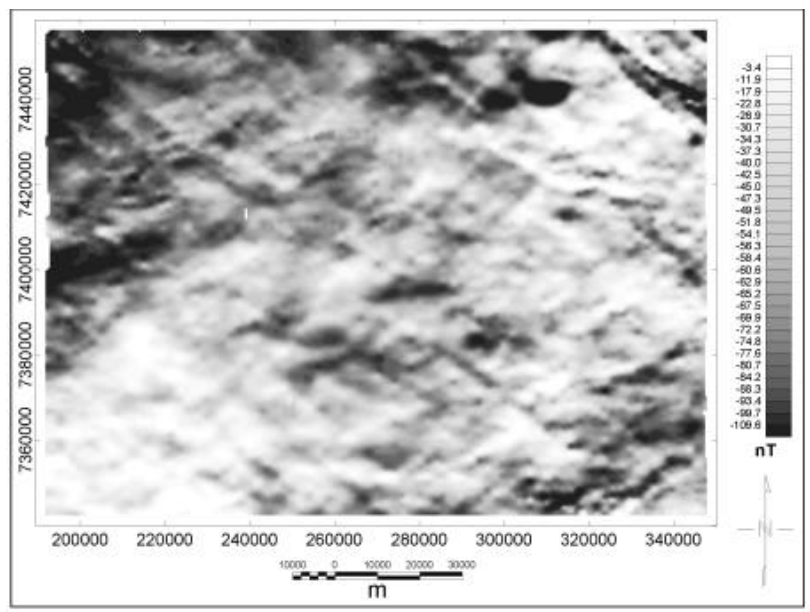

Figura 2 - Mapa magnético residual micronivelado através de decorrugação FFT.

Sobre os dados magnéticos mostrados na Figura 2 foi aplicado o método do sinal analítico (Nabighian 1972), baseado no cálculo da primeira derivada do campo magnético para as direções $\mathrm{X}, \mathrm{Y}$ e Z. A Figura 3 mostra o 
INTERPRETAÇÃO ESTRUTURAL-MAGNÉTICA DO EMBASAMENTO DA BACIA BAURU

mapa da amplitude do sinal analítico de ordem zero e o da fase do sinal analítico é exibido na Figura 4.

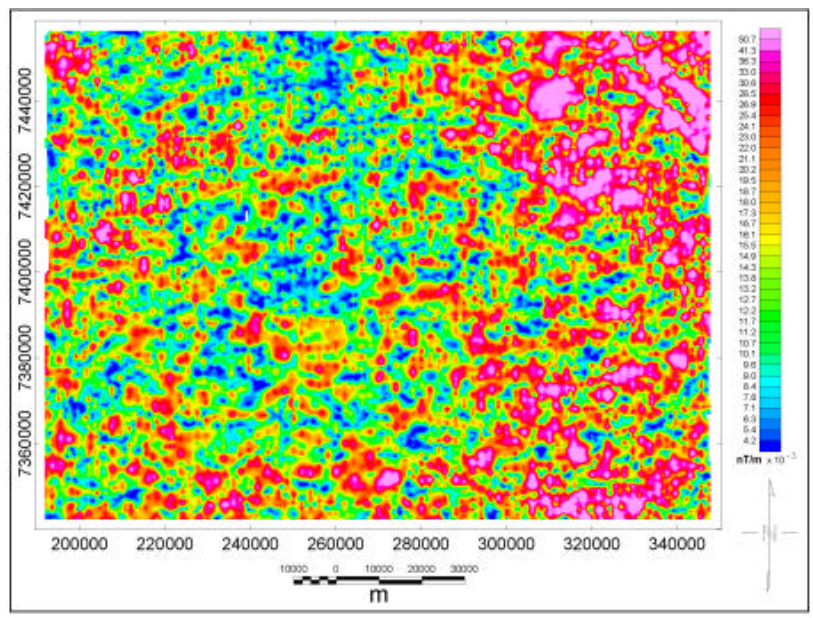

Figura 3 - Mapa da amplitude do sinal analítico de ordem zero.

Também foi gerado o mapa do gradiente horizontal de ordem zero (Milligan e Gunn 1997), com base nas derivadas do campo magnético para as direções $X$ e $Y$ (Figura 5).

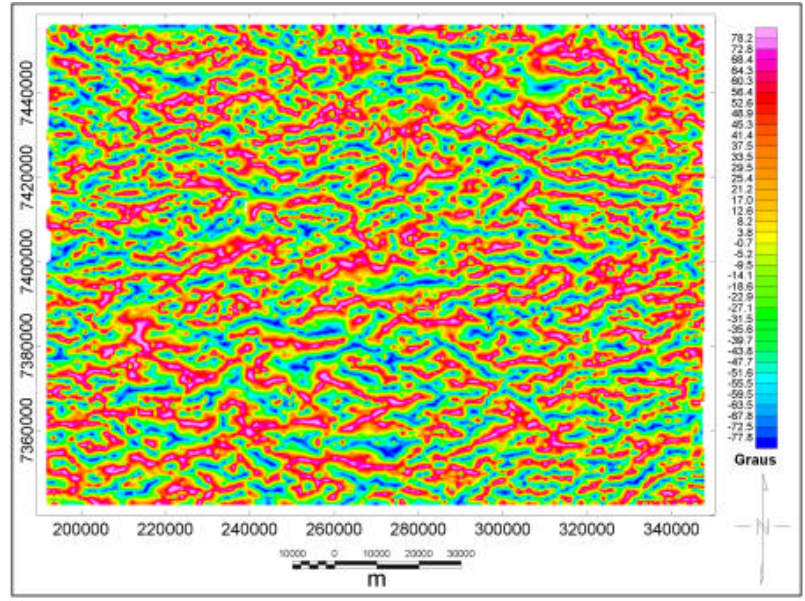

Figura 4- Mapa da fase do sinal analítico de ordem zero.

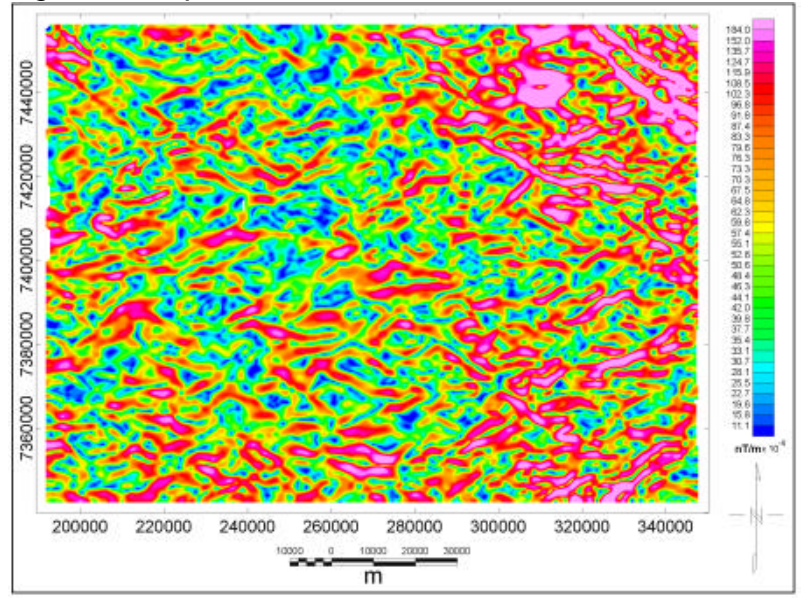

Figura 5 - Mapa do gradiente horizontal total de ordem zero.

\section{Resultados}

Através da integração dos alinhamentos magnéticoestruturais interpretados em cada um dos mapas gerados (figuras 3, 4 e 5), foi elaborado o mapa do arcabouço magnético-estrutural do embasamento da Bacia Bauru na área de estudo, mostrado na Figura 6, sobreposto ao mapa magnético residual. Neste mapa são mostrados os alinhamentos mais persistentes, identificados nos mapas das figuras 3,4 e 5 , correspondendo às principais estruturas das bacias sedimentares do Paraná e Bauru.

Visando a estimação da penetratividade destas estruturas na Bacia do Paraná foi aplicada, sobre o mapa magnético residual, a deconvolução de Euler, modelando as anomalias magnéticas com base no modelo geológico de dique, como esperado para o embasamento da Bacia Bauru. Objetivando a redução da quantidade das anomalias modeladas foram priorizadas as mais profundas, eliminando as fontes rasas e ruídos do sinal, através da aplicação da deconvolução de Euler aos dados magnéticos submetidos a continuação ascendente (Gunn 1975) para 2000 m (Figura 7).

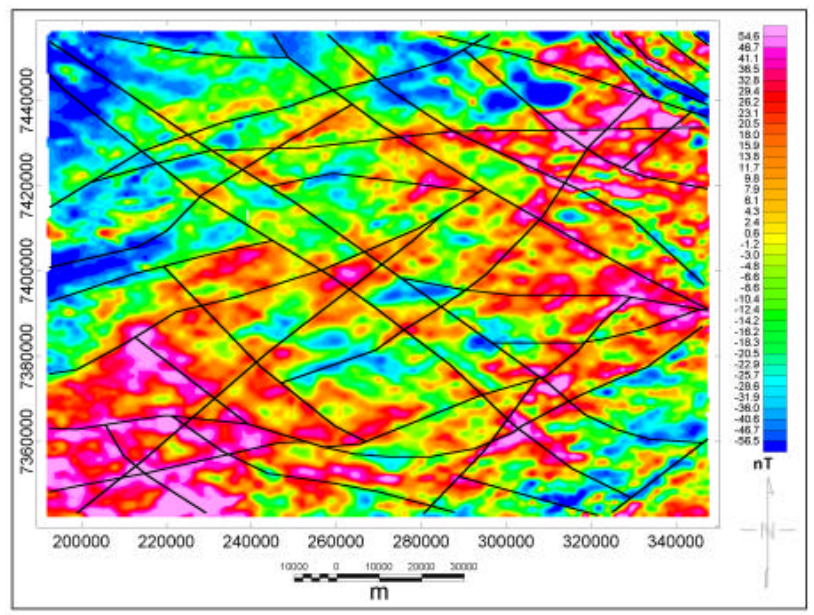

Figura 6 - Mapa do arcabouço estrutural-magnético do embasamento da Bacia Bauru, sobreposto ao mapa magnético residual.

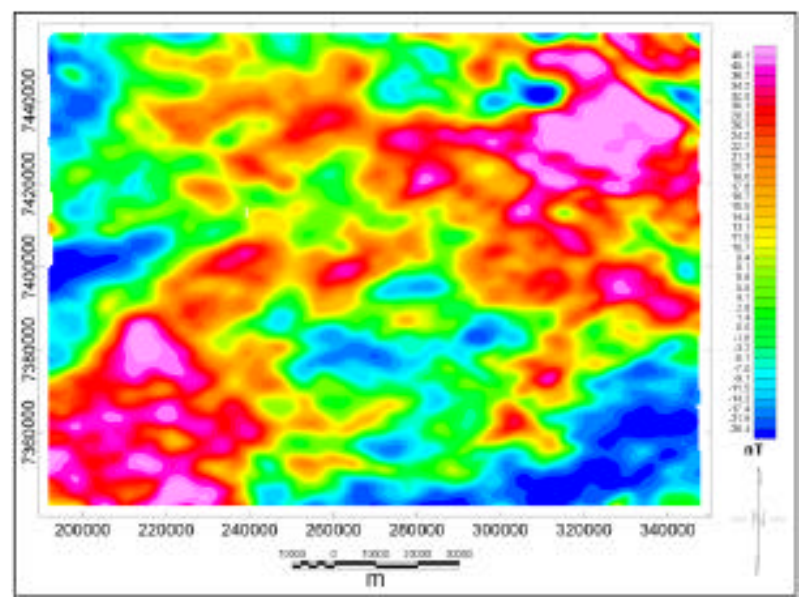

Figura 7 - Mapa magnético residual continuado para 2000 m. 
Daniel Fabian Bettú e Francisco José Fonseca Ferreira

O mapa de soluções de profundidades, provenientes da deconvolução de Euler, mostrado na Figura 8, foi construído utilizando uma janela de busca das anomalias com 10000 metros de largura (20 vezes o tamanho das células) e com o índice estrutural 1, referente ao modelo geológico de dique. É possivel perceber neste mapa que as fontes modeladas apresentam-se como pequenos segmentos alinhados, representando o enraizamento das estruturas no interior da Bacia do Paraná, conforme corrobora o histograma de frequência de classes de profundidades das fontes, referente ao mapa da Figura 8, mostrado na Figura 9. O histograma da Figura 9 exibe uma ampla distribuição de profundidades das fontes modeladas, com valores entre 750 e $4500 \mathrm{~m}$ de profundidade, e classe modal entre 2000 e $2250 \mathrm{~m}$, valores estes que exibem os melhores ajustes (alinhamentos) no mapa da Figura 8, indicando que as estruturas modelas possuem alta penetratividade nas rochas sedimentares da Bacia do Paraná.

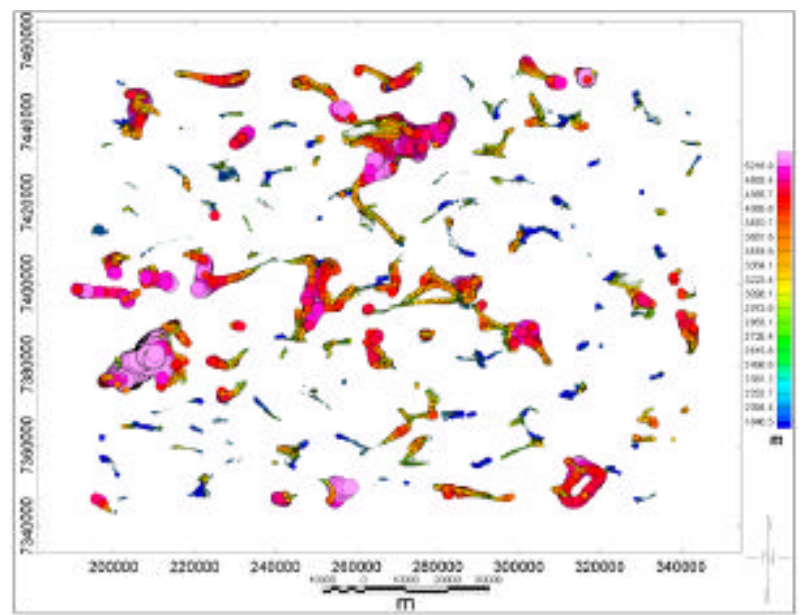

Figura 8 - Mapa de soluções de Euler para a continuação a 2000m, janela de busca de $10000 \mathrm{~m}$ e índice estrutural 1.

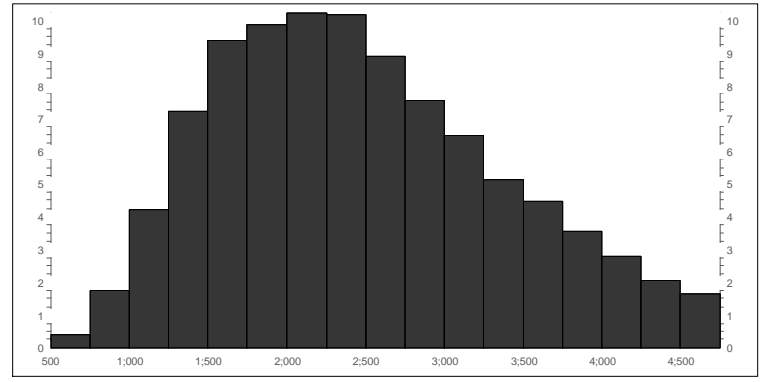

Figura 9 - Histograma de freqüência de soluções de profundidades de Euler para a continuação a $2000 \mathrm{~m}$ (janela de busca de $10000 \mathrm{~m}$ e índice estrutural 1).

Com base na interpretação integrada dos alinhamentos estruturais da superfície do Grupo Caiuá, extraídos do modelo numérico do terreno - MNT - (Figura 10) e da rede de drenagem da região (Figura 11) foi elaborado um modelo do arcabouço estrutural aflorante, exibido na Figura 12, sobreposto ao MNT.

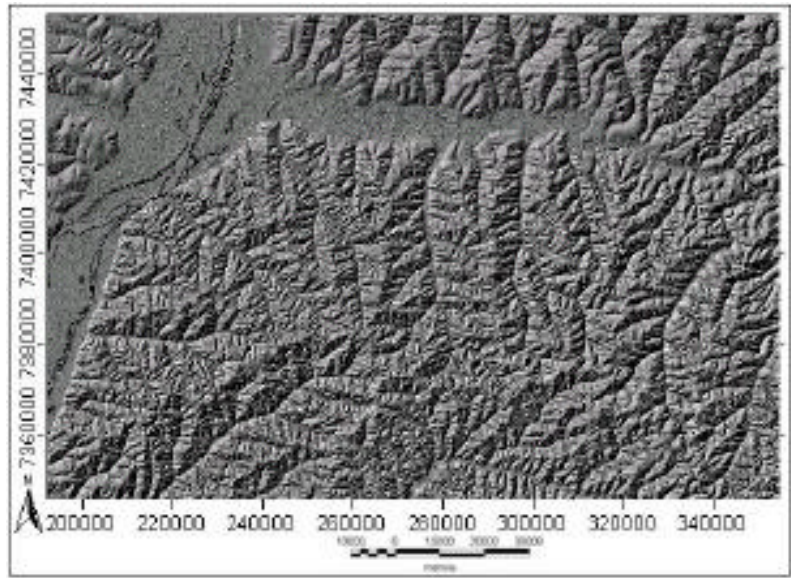

Figura 10. Modelo numérico do terreno.

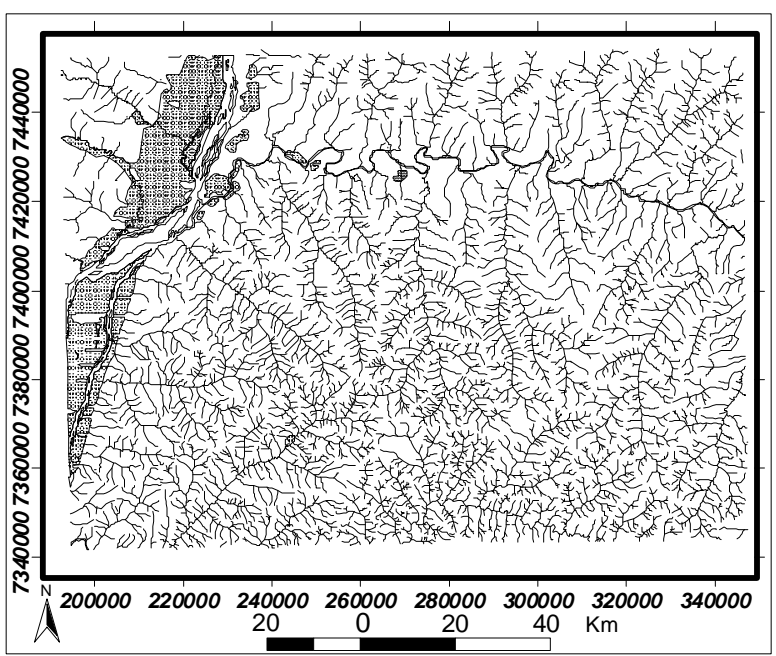

Figura 11 - Rede de drenagem da região.

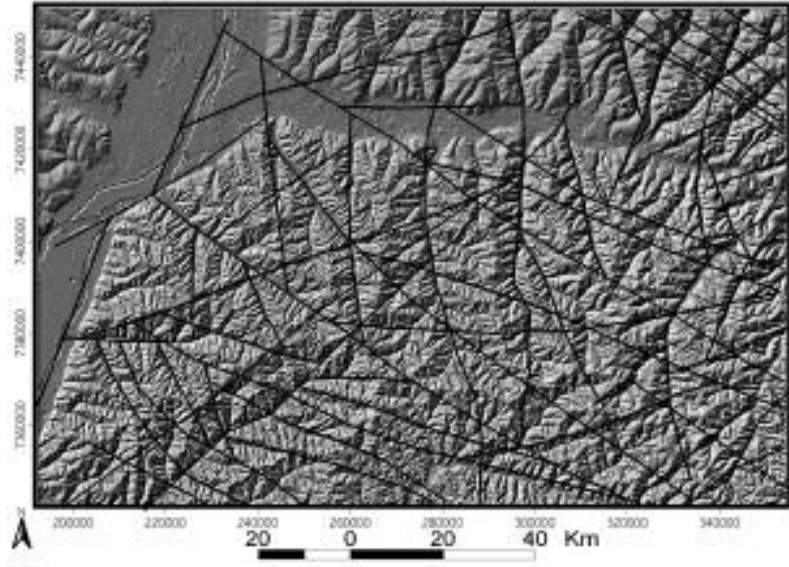

Figura 12 - Arcabouço estrutural da superfície dos arenitos do Grupo Caiuá. 
INTERPRETAÇÃO ESTRUTURAL-MAGNÉTICA DO EMBASAMENTO DA BACIA BAURU

A partir da integração dos modelos estruturais da superfície (Figura 12) e do embasamento da Bacia Bauru (Figura 6), visando identificar correspondência entre estruturas presentes nos dois mapas, foi gerado um mapa do arcabouço comum aos dois níveis de investigação, mostrado na Figura 13, sobreposto ao mapa magnético residual original.

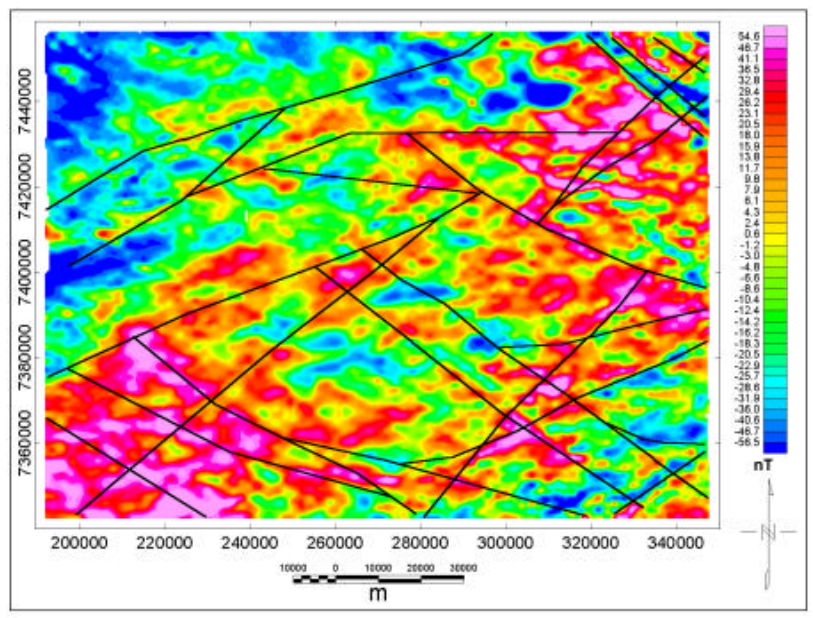

Figura 13 - Mapa do arcabouço estrutural comum à superfície e ao embasamento da Bacia Bauru, sobreposto ao mapa magnético residual original.

\section{Discussões e Conclusões}

O processamento dos dados magnéticos através da técnica do sinal analítico, e também do gradiente horizontal, mostrou-se bastante eficaz na interpretação dos alinhamentos magnético-estruturais, uma vez que ressaltou as principais feições anômalas presentes na área de estudo. $O$ alto contraste entre a susceptibilidade magnética dos arenitos e dos basaltos, bem como suas relações estratigráficas, favoreceram esta interpretação, permitindo a investigação magnética apenas do embasamento da Bacia Bauru.

A modelagem das anomalias magnéticas aplicada sobre o resultado da continuação ascendente dos dados para a altura de $2000 \mathrm{~m}$, através da deconvolução de Euler, resultou em uma importante suavização das feições anômalas, eliminando grande parte das fontes rasas e dos ruídos presentes no mapa magnético original. Este artifício permitiu a interpretação apenas das estruturas de maior magnitude na escala de trabalho adotada. $O$ resultado da modelagem mostrou que as estruturas identificas possuem alto grau de penetratividade na Bacia do Paraná, com a maioria das fontes situadas entre 2000 e $2250 \mathrm{~m}$ de profundidade.

A integração dos arcabouços estruturais do embasamento e da superfície da Bacia Bauru ressaltou a correspondência entre diversas estruturas presentes nos dois níveis de investigação, revelando a presença de relações genéticas entre elas. Desta forma ficou evidente a relação das grandes estruturas presentes na Bacia do Paraná, relacionadas muitas vezes a reativações de estruturas de seu embasamento, com aquelas identificadas na superfície dos arenitos do Grupo Caiuá, pertencentes àBacia Bauru.

\section{Agradecimentos}

Os autores agradem à Petrobrás pela cessão dos dados aerogeofísicos e ao Conselho Nacional de Desenvolvimento Científico e Tecnológico pela concessão de bolsa de mestrado, Fundo Setorial CTHIDRO, processo 132060/2002-7.

\section{Referências}

Briggs, I. C. 1974. Machine Contouring Using Minimum Curvature. Geophysics, 39(1): 39-48.

Fernandes, L. A. 1992. A Cobertura Cretácea Suprabasáltica no Paraná e Pontal do Paranapanema (SP): Os Grupos Bauru e Caiuá. São Paulo. 129 p. Dissertação de Mestrado, Instituto de Geociências, Universidade de São Paulo, Brasil.

Gunn, P. J. 1975. Linear Transformations of Gravity and Magnetics Fields. Geophysical Prospecting, 23: 300-312.

Milligan, P \& Gunn, P. J. 1997. Enhancement and Presentation of Airbone Geophysical Data. AGSO Journal of Australian Geology \& Geophysics, 17(2): 63-75.

Nabighian, M. N. 1972. The Analytic Signal of Twodimensional Magnetic Bodies with Poligonal Cross-section: Its Properties and Use for Autamated Anomaly Interpretation. Geophysics, 37: 507-517.

Petrobras, Petróleo Brasileiro S. A. 1980. Projeto Aerogeofísico Rio Ivaí: Consórcio CESP/IPT, Paulipetro (dados digitais). 